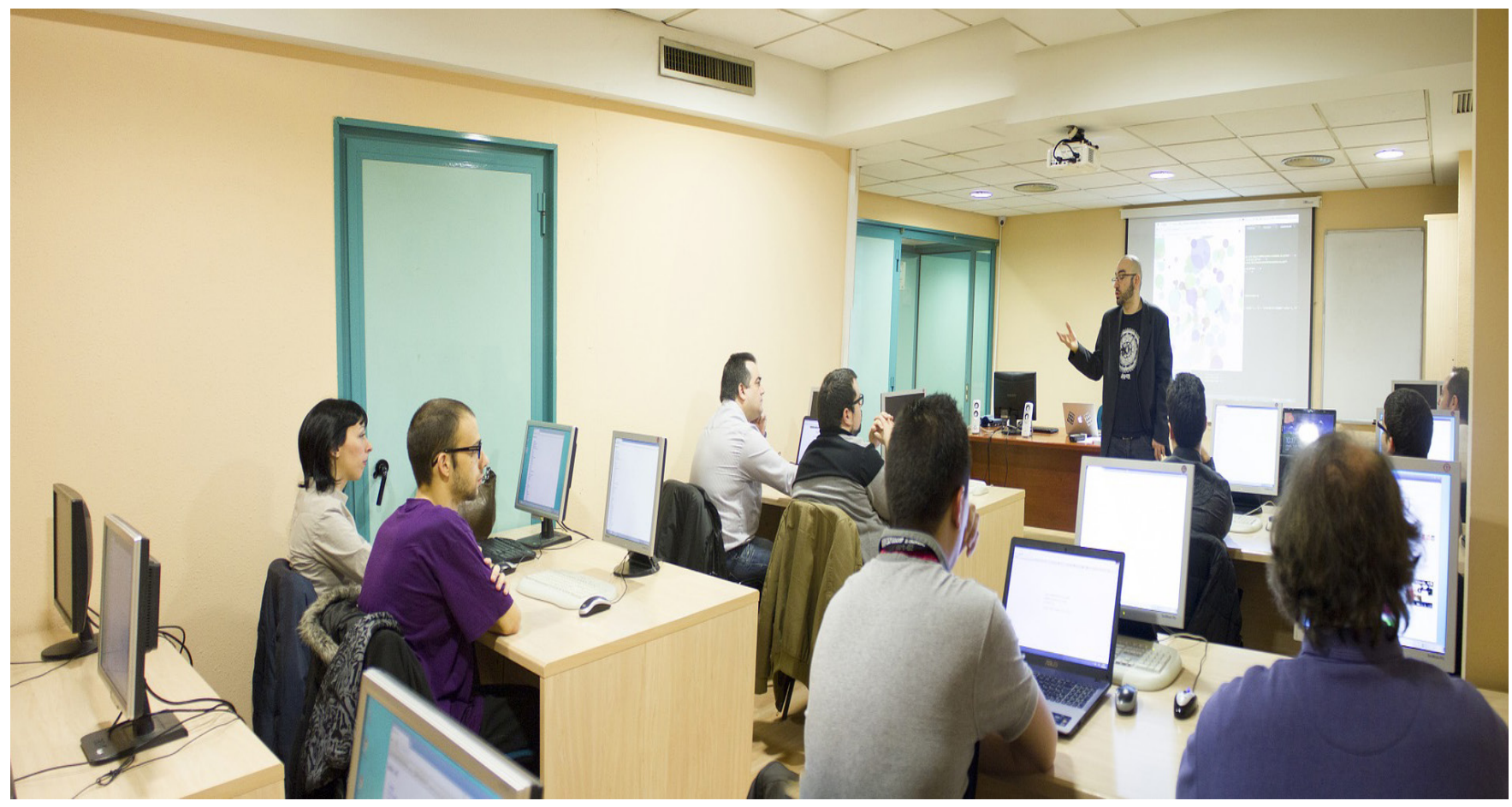

\title{
Incidencia de la Formación Pedagógica en la Práctica Docente
}

\section{Incidence of Pedagogical Training in Teaching Practice}

\section{Diana Gómez}

Estudiante semillerista, Diana.gomez@outlook.es, Fundación Tecnológica Antonio de Arévalo -TECNAR, Cartagena de Indias, Colombia

\section{Jorge Puello}

Estudiante semillerista, Jorge.puello@hotmail.com, Fundación Tecnológica Antonio de Arévalo -TECNAR, Cartagena de Indias, Colombia

\section{Sorely Soleno-Zambrano}

Estudiante semillerista, Sorely.soleno@gmail.com, Fundación Tecnológica Antonio de Arévalo -TECNAR, Cartagena de Indias, Colombia

Martha Benítez-Izquierdo

Especialista en Derecho Administrativo, matha.benitez@hotmail.com, Docente investigador. Fundación Tecnológica Antonio de Arévalo -TECNAR. Cartagena de Indias, Colombia 


\section{Resumen}

Este trabajo busca evidenciar la importancia de la formación pedagógica en la práctica docente de nuestros días mediatizado por las herramientas tecnológicas y acercamiento con el mundo productivo para propiciar mejores aptitudes y responsabilidades del estudiante como parte de un gran engranaje que es el proceso de enseñanza aprendizaje, evidenciando la importancia que tiene la constante actualización del docente y las consecuencias que pueda ocasionar el estancarse. La formación por competencias de la mano de las tecnologías de información e información es hoy día premisa para la evolución del capital intangible como lo es el conocimiento del ser humano; innumerables factores están puesto para que el aprendizaje ocurra, entre ellos se pueden conseguir, la vocación por enseñar el conocimientos, el compromiso abnegado y la motivación hacia los estudiantes en su carrera de estudio profesional, puesto que ellos son realmente los protagonistas del proceso de aprendizaje; por lo tanto, es sumamente importante la base pedagógica del profesorado especializado en las distintas carreras universitarias, ya que la misma le permitirá hacer uso de métodos que le permita a los alumnos conocer técnicas que puedan aplicar para resolver problemas reales en su cotidiano y en la sociedad.

Palabras clave: Formación, Pedagogía, Práctica, Docente.

\section{Abstract}

This work seeks to demonstrate the importance of pedagogical training in the teaching practice of our days mediated by technological tools and approach with the productive world to promote better skills and responsibilities of the student as part of a great gear that is the teaching-learning process, evidencing the importance of the constant updating of the teacher and the consequences that can cause stagnation. The formation by competences of the hand of the technologies of information and information is today premise for the evolution of the intangible capital as it is the knowledge of the human being; innumerable factors are set for learning to occur, among them can be achieved, the vocation to teach knowledge, selfless commitment and motivation towards students in their professional study career, since they are really the protagonists of the learning process; Therefore, the pedagogical basis of teachers specialized in the different university careers is extremely important, since it will allow you to use methods that allow students to know techniques that they can apply to solve real problems in their daily lives and in society.

Keywords: Training, Pedagogy, Practice, Teacher. 


\section{4}

\section{Introducción}

Este trabajo de aula vista como investigación documental busca evidenciar el rol que cumple el docente hoy en su práctica, basando aquel quehacer en su formación pedagógica como herramienta fundamental para generar la mejor adquisición de conocimientos y de competencias en todos sus niveles; siendo el estudiante el eje principal de formación, ya que este es el producto final del proceso de enseñanza aprendizaje, así refleja [1] "El estudiante es el centro donde convergen todas las acciones pedagógicas para lograr su formación integral y poder enfrentar las realidades complejas que se presentan en la contemporaneidad"

El mundo de hoy en día está cambiando y hay nuevas exigencias, así lo refleja en tanto [2] "un mundo altamente complejo como el actual expresa una profunda crisis", por lo tanto, surgen nuevas formas de producción y nuevas formas de hacer las cosas guiados por los elementos técnicos y tecnológicos que permiten que todo el paradigma del quehacer educativo cambie tras la implementación de nuevos métodos de enseñanzas y estrategias ligados a las herramientas tecnológicas y orientándolos más al mundo productivo tratando de acercar más al estudiante de su quehacer profesional.

Por lo anterior es importante indagar cuan preparado está en su formación pedagógica el docente para poder conseguir las competencias que el estudiante y/o alumno necesita saber, hacer y ser teniendo en cuenta la evolución de los tiempos y de las herramientas y métodos pedagógico actuales que le permitirán alcanzar los objetivos que se ha propuesto. Como señala [3-4] "un profesorado capacitado es el factor crítico para una mejor educación del alumnado".
Por consiguiente, este proyecto busca evidenciar la importancia de la formación pedagógica en la práctica docente de nuestros días globalizado por las herramientas tecnológicas de información y comunicación, innovando en las áreas del conocimiento, mostrando el acercamiento con el mundo productivo para propiciar mejores aptitudes $y$ responsabilidades del estudiante como parte de un gran engranaje que es el proceso de enseñanza aprendizaje. "Quienes apuesten por estas herramientas estarán en una mejor posición para lograr un lugar privilegiado en el futuro que hoy ya se empieza a diseñar" [5].

De acuerdo a al artículo El docente ante los retos educativos del siglo XXI de Griselda Vélez Chablé comenta "las instituciones educativas, y de manera especial las que imparten educación superior, deben participar en la formación de recursos humanos capaces de enfrentarse a los actuales cambios y responder así a las exigencias del mercado laboral y necesidades de la sociedad. Al respecto, la UNESCO destaca la importancia de que «las nuevas generaciones deberán estar preparadas con nuevas competencias y nuevos conocimientos e ideales» para la construcción del futuro" [6].

Es importante describir la problemática que se está dando mucho en las universidades y es la incursión de personal docente calificado en su área específica pero sin formación pedagógica lo que permite dificultades en la adquisición del conocimiento, manejos de estrategias de aprendizaje y por ende en los resultados de aprendizaje. esto se ve evidenciado en muchos artículos que muestran tal preocupación como lo es el caso de la universidad de la sabana que plasma lo siguiente "si bien es cierto que el ejercicio de la enseñanza tiene mucho de conocimiento práctico, es decir, de experiencia, también lo es que esta experiencia se desarrolla y acumula con mayor facilidad y acierto si cuenta con 
adecuados soportes teóricos e instrumentales, que son susceptibles de ser aprendidos por los profesores de manera simultánea con el desarrollo de su actividad educativa: es lo que podemos llamar formación en servicio o formación continua [7]. La formación de este tipo es la más común en la docencia universitaria en Colombia, pues, salvo en las facultades de educación, no es frecuente que los profesores universitarios tengan una formación pedagógica previa a su vinculación como docentes.

De igual manera, a nivel internacional también hay la misma preocupación por parte de [8], quien comenta lo siguiente "La Educación Superior se enfrenta a una serie de desafíos en un mundo que se transforma, por ello debe revisar su misión y redefinir muchas de sus tareas sustantivas, en especial aquellas que se relacionen con las necesidades de la sociedad en materia de aprendizaje y superación continua. Un punto clave en su misión está dirigido a destacar las tareas de las universidades, Se requiere llevar a cabo esfuerzos para elevar la formación pedagógica de los profesores, lo cual tributará en una mejor preparación de los egresados universitarios", por lo tanto, es de suma importancia cultivar nuestro conocimientos y afianzarlos en una constante actualización de competencias profesionales.

Este trabajo de aula se enmarca dentro de un análisis importante porque permite observar el nivel de formación pedagógica de los docentes encargados de egresar personas a la vida profesional y laboral permitiendo destacarse en las actividades propias de su ejercicio teniendo en cuenta que la actividad académica va cambiando con los tiempos y las estrategias y metodologías deben ir acordes con las herramientas técnicas y tecnológicas que tengamos a nuestro servicio. De ahí la importancia de la formación pedagógica en la práctica docente que le permita al mismo potenciar tu actividad de enseñanzaaprendizaje a través de los medios que ésta permita, por ello se repondenderá en el presente trabajo a la siguiente pregunta ¿Cuál es la incidencia de la formación pedagógica en la práctica docente? y por ello el alcance pretende describir la incidencia de la formación pedagógica en la práctica Docente.

\section{Marco Teórico}

"La administración educativa busca reconocer los méritos docentes, incentivar la carrera profesional, pero también crear un registro de docentes para puestos de desempeño determinados" [9-10].

Teóricamente hablando los conceptos de pedagogía y docencia son muy reconocidos y son de mucha importancia dentro del entorno escolar, educación media, media técnica, educación para el trabajo y educación superior. Siempre donde exista un deseo de aprender y quien enseña se generará la relación estudiante - profesor y de allí parte la importancia de este tema de investigación, asi lo refleja [11] "La Pedagogía es tarea de los formadores de maestros interpretar esta coyuntura y orientar a los futuros docentes hacia la reflexividad crítica que debe caracterizar a todo maestro, y también la definición de la identidad profesional del maestro, como pedagogo, como experto de la educación, ya que ese es su campo de acción, no es la sicología, no es la administración, ni mucho menos la calidad".

Del mismo modo, [12], sostiene que "La pedagogía a lo largo de su historia ha sufrido grandes cambios y ha pasado por diversas formas de ser asumida, desde ser vista como arte, como disciplina, como ciencia o filosofía de la educación; esto de acuerdo a la época en la cual surge el pensamiento en torno a ella"; cabe anotar, que es importante reconocer el papel de los profesores en sus prácticas como acompañantes, más aún, 


\section{6}

cuando tienen la disponibilidad de tiempo para atender a las necesidades que requieren los estudiantes, puesto que es un compromiso que permite ir en búsqueda del éxito parcial de los procesos y momentos en los que se trabaja las diferentes temáticas y aprendizajes, y para que estos aprendizajes sean significativos en función de las dificultades conceptuales entre la metodología y la didáctica, por supuesto, sin confundirlas con las posibilidades y los contextos [13].

En el mismo orden de ideas, de acuerdo a lo vertiginoso del mundo siempre en busca de innovar y estar actualizado, de acuerdo a esta premisa cabe resaltar la importancia que le da la autora a mantenerse actualizado en todo el contexto educativo incluyendo, herramientas, modelos, métodos de enseñanza y las TIC'S [14-15] como nuevo aspecto inmerso dentro de los nuevos roles que debe ejercer el docente en su práctica, el manejo y uso de las Tecnologías de la información y la comunicación como mecanismo que impulsa y desarrolla las actividades de enseñanza - aprendizaje del aula de manera directa o indirecta como parte del trabajo individual del estudiante que le permite potencializar sus conocimientos, habilidades y destrezas dentro del proceso educativo. "Quienes apuesten por estas herramientas estarán en una mejor posición para lograr un lugar privilegiado en el futuro que hoy ya se empieza a diseñar" [5].

De acuerdo a las anteriores apreciaciones vale la pena resaltar cual debe ser la formación pedagógica ya vista meramente para un profesor / docente universitario contando que es un proceso de enseñanza -aprendizaje diferente con actores inmersos dentro de la actividad, con conocimientos previos muy diferentes a los de la educación media y para el trabajo y desarrollo humano; para lo cual se soporta este interrogante en lo planteado por [7]" quien expresa que "Se concibe la formación pedagógica del profesorado universitario como un proceso continuo que atendiendo a diferentes etapas organizadas en su práctica docente, facilitan iniciar, adiestrar, formar y perfeccionar a dichos profesores en el dominio de los contenidos de la didáctica de la educación superior con el propósito de incidir en la calidad de la formación de los estudiantes lo que influye en la calidad de la educación superior.

\section{Materiales y métodos}

El presente estudio es de tipo cualitativo que permite recolectar información para su respectivo estudio; de igual manera, será de carácter descriptiva la cual busca describir situaciones que nos acerquen más a la resolución del objeto de estudio, así mismo está apoyada en una revisión documental que por lo tanto, va a proveer de un análisis riguroso de los con el fin de generar unas conclusiones que permitan aclarar la relevancia del objeto de estudio como las diferentes estrategias y actividades que se han hecho en pro de la formación pedagógica en la práctica docente y cuáles son las repercusiones de su carencia en la misma.

Fuentes de información.

La fuente de información utilizada para este trabajo fue la secundaria que es aquella información primaria, sintetizada y reorganizada. Están especialmente diseñadas para facilitar y maximizar el acceso a las fuentes primarias 0 a sus contenidos. Componen la colección de referencia de la biblioteca y facilitan el control y el acceso a las fuentes primarias.

\section{Resultados}

A través de todo este recorrido se ha podido entender de la importancia que tiene para las Instituciones de Educación Superior impartir una educación de calidad partiendo 
principalmente de un talento humano efectivo que propenda por la formación $y$ actualización constante en su área disciplinar y principalmente en el área de la pedagogía y de su práctica docente, así lo refleja [16] se deben "desarrollar y fortalecer una competencia inclusiva y de interculturalidad en la formación y ejercicio docente contribuiría de manera concreta y concisa, entre otras políticas y acciones que deben implementarse y fortalecerse, al logro de la dimensión social de la educación superior". como resultado de esta afirmación se puede evidenciar artículos emanados del Ministerio de Educación Nacional en la que emite información alusiva a la educación "El Ministerio de educación ha desarrollado propuestas de formación tendientes a acompañar de manera diferenciada a las secretarías de educación en su gestión para la definición de planes de apoyo coherentes, pertinentes y viables, que apunten a la definición y desarrollo de programas y proyectos para el mejoramiento educativo en distintos ámbitos, tales como: el uso y apropiación de medios y tecnologías de la información y la comunicación, fortalecimiento de la gestión escolar y formación de docentes y directivos docentes.

Cómo se puede ver en el párrafo anterior desde el MEN (Ministerio de Educación Nacional), No sólo hay preocupación por este objeto de estudio sino que además se tienen propuestas para poder contrarrestar esta situación que iría en detrimento de la calidad en la educación y en la formación de los estudiantes en todos sus niveles. Vale la pena resaltar sus esfuerzos por brindarle a los docentes de la educación superior las herramientas necesarias para actualizarse en manejo de las herramientas TIC'S como también en el desarrollo de su práctica docente para lo cual la autora [5] expresa que "la Educación Superior se enfrenta a una serie de desafíos en un mundo que se transforma, por ello debe revisar su misión y redefinir muchas de sus tareas sustantivas, en especial aquellas que se relacionen con las necesidades de la sociedad en materia de aprendizaje y superación continua.

La actividad del profesor ha sido y seguirá siendo un aspecto de estudio de la Didáctica cada vezes más evidente su papel de facilitador en la calidad del proceso de enseñanza aprendizaje y en la educación en general, para el cual puede apoyarse en herramientas didácticas que atraigan a los estudiantes y les genere curiosidad para que lleguen a ser exitosos en su desempeño laborar [17]. Así lo refleja [18] "la formación docente, se entiende como la "acción profunda ejercida sobre el sujeto, tendiente a la transformación de todo su ser, ocupando una posición intermedia entre educación e instrucción que posibilita la transformación del sujeto que se forma en los planos cognoscitivos, afectivos y sociales, orientando el proceso mediante una lógica de estructuración y no de acumulación".

La mejora de la calidad del proceso de enseñanza aprendizaje pasa necesariamente por la transformación del pensamiento y de los sentimientos de los profesores, para ello la Educación Superior necesita de la calidad del personal docente, de los programas y de los estudiantes, de las infraestructuras y del ambiente universitario", de igual modo, [19] que la pedagogía es una ciencia o disciplina a la manera de las ciencias humanas y que, por tanto, no reconocer esta forma de existencia de la pedagogía es desconocer, de igual modo, la forma de existencia de otras disciplinas que también son ciencias humanas, desconocimiento que hace del crítico de su propuesta un positivista a ultranza.

En términos generales todas las propuestas en especial la del Sistema Colombiano de Formación y desarrollo profesional docente busca asumir las realidades de la formación docente en nuestro país, para emprender caminos de articulación de acciones 


\section{8}

tendientes a transformar las situaciones polémicas, consolidar los avances de los planes, programas, proyectos y estrategias exitosas para avanzar en el la búsqueda de alternativas de formación docente que incidan de manera pertinente y eficaz en la institución escolar. Según menciona [20], un programa formativo destinado al profesorado tendría entre sus implicaciones básicas una trayectoria de formación que diferencia tres momentos esenciales: de formación inicial, de introducción al ejercicio profesional y un momento continuo de desarrollo profesional que incluyera instancias formales e informales de formación.

Se trata de formar docentes para asumir el compromiso de educar a las nuevas generaciones en procesos de aprendizaje de calidad que les permitan participar competentemente en las distintas esferas de la actividad social y hacer realidad el principio de la educación como derecho para todos, no sólo a través del acceso al sistema educativo sino por los procesos formativos que en él se desarrollan y la calidad de los aprendizajes escolares. Según menciona [19], un programa formativo destinado al profesorado tendría entre sus implicaciones básicas una trayectoria de formación que diferencia tres momentos esenciales: de formación inicial, de introducción al ejercicio profesional y un momento continuo de desarrollo profesional que incluyera instancias formales e informales de formación.

\section{Conclusiones}

Este proyecto es materia primera para observar cuales son aquellas debilidades o situaciones problemáticas de la práctica docente llevada a cabo sin la formación pedagógica que ésta amerita, siendo importante el diagnóstico de la formación disciplinar y pedagógica del personal docente para orientar los esfuerzo de cualificación docente a la sensibilización, $y$ toma de conciencia por parte de docentes y directivos de la gran responsabilidad que se tiene con estudiantes al momento de orientar procesos de enseñanza aprendizaje guiados por buenas prácticas de metodologías, de la didáctica, de herramientas de las Tics y de todos aquellos aspectos que permiten que la relación académica con estudiantes lleguen a dar los resultados esperados y que la calidad del egresado sea la más óptima para desenvolverse en la vida social y ser útil para una sociedad cada vez más exigente, competitiva y de la información y la comunicación.

\section{Referencias}

[1] E. Barrientos A. Suárez, L. Fajardo y Y. Ríos "Estrategias pedagógicas de micro finanzas a los estudiantes del Colegio Municipal Aeropuerto", Revista Convicciones, no. 08, pp. 17-21, 2017

[2] J. Monsalve y E. Barrientos, "Formación para la gerencia del docente en la promoción y desarrollo de una cultura ambientalista", Mundo Fesc, vol. 7, no. 13, 2017

[3] A. Bolivar, "¿Dónde situar los esfuerzos de mejora?: política educativa escuela y aula", educaçao \& sociedade, vol. 26, no. 92, pp. 859888,2005

[4] L. Montañez Carrillo y J.P. Lis Gutiérrez, "Medición de la Madurez de la Gestión del Conocimiento en la Escuela de Ciencias Básicas Tecnología e Ingeniería de la UNAD", Publicaciones E Investigación, vol. 10, 177191, 2016

[5] J. Spector, "Emerging Educational Technologies and Sesearch Directions", Journal of educational technology \& society, vol. 16, no. 2, april, 21-30, 2013

[6]F.Villalpando,Eldocente antelosretoseducativos 
del siglo XXI, 2018. [En línea]. Disponible en: https://issuu.com/fatimavillalpando/docs/el docente_ante_los_retos_educativo

[7] M. Álvarez, "La relación teoría-práctica y el desarrollo profesional docente: obstáculos y retos", Educere, vol. 19, no. 63, 2015

[8] M. Cáceres Mesa, L. Lara Díaz, C. Miriam Iglesias León, R. García Cruz, G. Bravo López, C. Cañedo Iglesias y O. Valdés Chaviano, "La formación pedagógica de los profesores universitarios: Una propuesta en el proceso de profesionalización del docente", Revista Iberoamericana de Educación, no. 33, 2003

[9] M. Alfageme, J. Arencibia y A. Guarro A, "Contenidos e incidencia en la práctica de la formación docente en canarias". Profesorado. Revista de currículum y formación de profesorado, vol. 20, no. 2, 2016

[10] J.A. Parada Puentes, "Estrategias Gerenciales para el Reconocimiento del Desempeño Laboral Docente", Mundo FESC, vol. 7, no. 14, pp. 42$56,2018$.

[11] J. Cossio, "Pedagogía y calidad de la educación: una mirada a la formación del maestro rural", Sophia, vol. 10, no. 1, pp. 14-23, 2014

[12] J. Orrego, "La pedagogía como reflexión del ser en la educación", Revista latinoamericana de estudios educativos (colombia), vol. 3 , no. 1 , pp. 27-39, 2007

[13] C. García-Quintero y G. Villamizar Suárez, "Análisis fenomenológico de la conciencia del docente a partir de sus prácticas evaluativas", Revista Perspectivas, vol. 2, no. 2, pp. 49-59, 2017

[14] M.M. Martín, C.A. Hernández-Suarez y S.M. Mendoza-Lizcano, "Ambientes de aprendizaje basados en herramientas web para el desarrollo de competencias TIC en la docencia", Revista Perspectivas, 2(1), 97-104, 2017

[15] J. Hernández-Sánchez, E. Borjón-Robles y M. Torres-Ibarra, "Dimensiones de la tecnología en la formación inicial de profesores de matemáticas: un estudio desde el currículum oficial", Ecomatemático, vol. 7, no. 1, pp. 6-19, 2016

[16] D. Montes y C.I. Suárez, "La formación docente universitaria: claves formativas de universidades españolas", Revista electrónica de investigación educativa, vol. 18, no. 3, pp. 51-64, 2016

[17] T. Velásquez Pérez, E.E. Espinel Blanco y G. Guerrero Gómez, "Estrategias pedagógicas en el aula de clase", Revista Colombiana de Tecnologías de Avanzada, vol. 2, no. 28, 2016

[18] I. Gorodokin, La Formación docente y su relación con la epistemología. San Luis, Argentina: Universidad Nacional - Escuela Normal Juan Pascual Pringues. 2002

[19] R. Flórez, "El fin de la historia o el fin de la pedagogía”, Cuadernos Pedagógicos, no. 2, pp. 17 - 22, 1998

[20] J. Tejada, "Profesionalización docente en la universidad: implicaciones desde la formación", Revista de Universidad y Sociedad del Conocimiento, vol. 10, no. 1, pp. 170-183, 2013 